\title{
COMMENTARY
}

\section{Beyond Stiffness}

\section{Collagen Signaling in Pancreatic Cancer and Pancreas Regeneration}

\author{
Huocong Huang* and Rolf A. Brekken ${ }^{\star \dagger}$
}

From the Division of Surgical Oncology, * Department of Surgery, and Hamon Center for Therapeutic Oncology Research, and the Department of

Pharmacology, ${ }^{\dagger}$ University of Texas Southwestern Medical Center, Dallas, Texas

Pancreatic ductal adenocarcinoma (PDA) is projected to be the second leading cause of cancer-associated deaths in the United States by $2030 .^{1}$ The dismal prognosis of PDA patients is due to early dissemination of the tumor, therapy resistance, and the fact that most patients present with locally advanced or metastatic disease at diagnosis. ${ }^{2}$ Thus, a small percentage of patients are candidates for surgical resection, the current most effective therapy. PDA patients receive chemotherapy and radiotherapy as standard treatment, which often renders limited improvement of prognosis; as a result, the current overall 5-year survival rate after PDA diagnosis is approximately $10 \%$.

PDA is characterized by a prominent stroma, composed of a wide variety of nonneoplastic cells, including cancerassociated fibroblasts, myeloid cells, lymphocytes, and vascular endothelial cells, as well as marked extracellular matrix (ECM) deposition. ${ }^{3}$ This complex fibrotic network often constitutes up to $80 \%$ to $90 \%$ of the tumor volume and has important functions in PDA progression, including tumor growth, invasion, metastasis, immune evasion, and therapy resistance. For example, as a result of extensive ECM deposition, interstitial fluid pressure is elevated within the tumor, which impairs perfusion and drug delivery. ${ }^{4} \mathrm{ECM}$ is also considered a barrier for T-cell infiltration, resulting in a significant fraction of PDA being T-cell deficient. ${ }^{5}$ In addition, the ECM also promotes tumor progression directly through ECM-activated signaling pathways on tumor cells. ${ }^{6}$ Despite the observation that ECM signaling can support PDA progression, multiple studies have reported that high stromal content of PDA correlates with a more favorable outcome in PDA patients. ${ }^{7,8}$ Furthermore, the depletion of ECM-producing cancer-associated fibroblasts in robust preclinical models resulted in a more aggressive PDA phenotype and reduced survival. ${ }^{9}$ These contrasting data suggest that the stroma may also have tumor-restricting function and underline the complexity of ECM biology in PDA. Therefore, it is important to understand the function of specific ECM components and the signaling pathways they activate.

Collagens are the most abundant ECM protein in PDA stroma. Collagens function as major structural components of the ECM and interact with cells through cell surface receptors. There are four major classes of collagen receptors: integrins, discoidin domain receptors (DDRs), glycoprotein VI, and leukocyte-associated Ig-like receptor-1. ${ }^{10}$ Among these collagen receptors, DDRs are unique in that they are receptor tyrosine kinases. DDRs contain an $\mathrm{N}$-terminal extracellular discoidin domain containing a collagen binding site. There are two types of DDRs: DDR1 and DDR2. Expression of DDR1 and DDR2 can overlap, but generally DDR1 is expressed by epithelial cells, whereas DDR2 is expressed in cells of mesenchymal origin. DDRs are important for physiological development, as evidenced by the phenotype of $D d r 1$ - or $D d r 2$ deficient animals, which includes smaller organ size, reduced bone growth, and defects in lactation and embryo implantation. ${ }^{11,12}$ Yet, the tissue-specific functions of DDRs are not

Supported by NIH grant U54 CA210181 project 2, the Effie Marie Cain Fellowship, and the Jean Shelby Fund for Cancer Research at Communities Foundation of Texas.

Accepted for publication May 6, 2020.

Disclosures: None declared.

Address correspondence to Rolf A. Brekken, Ph.D., Hamon Center for Therapeutic Oncology Research, University of Texas Southwestern Medical Center, 6000 Harry Hines Blvd., Dallas, TX 75390-8593. E-mail: rolf. brekken@utsouthwestern.edu 
fully understood. More important, considering the abundance of collagens in the stroma of different cancer types, especially PDA, identifying the contribution of DDRs to tumor progression is critical and potentially an untapped therapeutic avenue.

In an effort to understand the contribution of DDR1 in pancreas remodeling and PDA progression, Ruggeri et al, ${ }^{13}$ in this issue for the first time genetically ablated $D d r l$ in $K P C$ mice $\left(\mathrm{Kras}^{\mathrm{Gl2D} /+}\right.$; $\operatorname{Trp} 53^{\mathrm{RI72H/+}}$; Ptfla $\left.{ }^{\mathrm{Cre} /+}\right)$, which is a genetically engineered mouse model widely used to recapitulate the progression of PDA. Ruggeri et $\mathrm{al}^{13}$ found that $D d r l$ was expressed highly in PDA, and strikingly, $D d r l$-null $K P C$ mice developed well-differentiated, less proliferative, and poorly metastatic tumors compared with control Ddrl-wildtype $K P C$ mice. These data are consistent with previous studies from our group and others, suggesting that DDR1 functions to promote PDA progression. For example, collagen signaling through DDR1 can drive tumor cell plasticity. ${ }^{14,15}$ Consistent with this, pharmacologic inhibition of DDR1 significantly reduces PDA tumor growth, reduces cancer cell invasiveness, and improves chemotherapy response in vivo. ${ }^{16,17}$ DDR1 activity has also been implicated in driving tumor progression in other cancer types. For instance, genetic and pharmacologic inhibition of DDR1 reduced tumor initiation and tumor progression in KRAS-mutant lung adenocarcinoma, and the combinatory inhibition of DDR1 and Notch signaling induced the regression of $K R A S$-mutant patient-derived lung xenografts with a therapeutic efficacy that was favorable compared with standard chemotherapy. ${ }^{18}$ However, in breast cancer, the function of DDR 1 is more complex. An earlier study found that DDR1 signaling enables breast cancer cells to undergo metastatic reactivation in multiple target organs, ${ }^{19}$ whereas a more recent study genetically ablated $D d r l$ in $M M T V-P y M T$ mice and found that $D d r l$-null mammary tumors grew faster, featured by a more basal phenotype with increased epithelial tension, matricellular fibrosis, and lung metastases. ${ }^{20}$

Therefore, the function of DDR1 appears to be cancer type or microenvironment dependent. This might be due to cell lineage differences of DDR1-expressing cells in different cancer types. How DDR $1^{+}$cells contribute to the progression of cancer might determine the outcome of DDR1 targeting. For instance, in the MMTV-PyMT model, Ddrl was found to be expressed by luminal cells but not myoepithelial cells, and deletion of $D d r l$ induced basal differentiation of $\mathrm{CD} 90^{+} \mathrm{CD} 24^{+}$cancer cells, resulting in the increase of mitotic basal cells. ${ }^{20}$ Intriguingly, an unbiased bioinformatics study based on large human genomic and transcriptomic data sets reported that $D D R 1$ expression is significantly enriched in a small subset of drug-resistant aldehyde dehydrogenase-positive $\left(\mathrm{ALDH}^{+}\right)$stem-like cancer cells in breast cancer, independent of cancer molecular subtype. ${ }^{21}$ This report suggests that targeting DDR1 remains an attractive goal in breast cancer. In $K R A S$-mutant lung cancer, DDR1 was also found to be expressed by a subset of cancer cells exhibiting an aggressive transcriptional profile, although cell lineage was not specifically identified. ${ }^{18}$ Although Ruggeri et al ${ }^{13}$ found that Ddrl deletion strikingly inhibited $K P C$ tumor progression, deletion of $D d r l$ did not improve survival because loss of $D d r l$ also induced severe pancreatic atrophy. As a result, the animals died from pancreatic insufficiency. This strongly suggests that DDR1 is expressed by a subset of cells that can regenerate exocrine pancreas function during tissue damage. To further investigate the function of $D d r l$ at an earlier stage of PDA tumorigenesis, Ruggeri et al ${ }^{13}$ crossed $D d r 1$-null mice into $K C$ $\left(\mathrm{Kras}^{\mathrm{Gl2D/+}} ; \mathrm{Ptfla}^{\mathrm{Cre} /+}\right)$ animals and treated these mice with cerulean to induce experimental pancreatitis. Interestingly, $D d r l$ was expressed at a low level in normal pancreas but was markedly up-regulated during tissue damage caused by pancreatitis or neoplastic lesions. Compared with control mice, the pancreatic acinar cell population recovered more slowly in Ddrl-null mice, due to a delay in epithelial cell proliferation. As a result, $D d r 1$-null mice failed to resolve tissue damage at 6 weeks after cerulean treatment, resulting in a dramatic decrease of pancreatic acinar tissue mass.

Ruggeri et $\mathrm{al}^{13}$ hypothesized that the delay in damage recovery was due to a disruption in the regeneration potential of acinar cells. They investigated a Stathmin1-positive acinar cell population, which is a recently identified functionally and molecularly distinct acinar subpopulation with progenitor properties. ${ }^{22}$ Following chemical-induced pancreatitis, these cells proliferate to recover the injured acinar tissue. At baseline, control and Ddrl-null mice had similar numbers of Stathmin 1positive cells. After injury, control mice showed a large expansion of Stathmin1-positive, proliferating cell nuclear antigen-positive cells; however, the expansion was substantially delayed and quickly subsided in $D d r l$-null mice. These data strongly suggest that DDR1 is critical for acinar progenitor expansion to regenerate the injured pancreas. This is relevant to the observation that DDR1 enhances PDA cancer cell plasticity and resistance to chemotherapy. It is important for future studies to determine the function of Stathmin1positive cells during PDA progression and whether these cells have cancer stem cell properties; in addition, it is also important to determine whether DDR1 contributes to stemness and the expansion of this cancer cell population that leads to tumor aggressiveness and therapy resistance.

Another interesting phenotype of the Ddrl-null KPC mice is increased fibrosis, which was also observed in $D d r l$-null MMTV-PyMT tumors. ${ }^{20}$ This might be a compensatory response due to the failure of injured tissue regeneration. Consistent with these observations, the total number of fibroblasts increased in Ddrl-null pancreas during recovery following cerulean treatment. However, because Ddrl was deleted globally in this study, it is difficult to determine whether this fibrotic reaction was induced by paracrine signals indirectly or a direct effect of $D d r l$ ablation in fibroblast progenitors. A conditional $D d r l$ deletion model in a cell type-specific manner may help address this question. More important, DDR1 is a well-appreciated contributor to the fibrotic reaction and collagen remodeling in other fibrotic diseases, such as pulmonary fibrosis and renal fibrosis. ${ }^{23,24}$ Recent advances in single-cell transcriptomic technologies will enable the identification of DDR1-expressing cells 
in different organs and tissues, to better understand the cell-specific function of DDR1 involved in tumorigenesis and fibrosis in the future.

Taken together, this study from Ruggeri et $\mathrm{al}^{13}$ illuminates the important function of DDR1 during pancreatic acinar tissue regeneration and PDA development. The extensive desmoplasia and high expression of DDR1 in PDA strongly suggest that it is a potential therapeutic target in desmoplastic tumors, such as PDA. Although orally available DDR1-specific inhibitors are available, ${ }^{17,25}$ their chronic use should be recommended with the knowledge that genetic ablation of $D D R I$ can cause acinar atrophy and pancreatic insufficiency. Pancreatic insufficiency can be mitigated, at least temporarily, by supplementation with dietary pancreatic enzymes. ${ }^{26}$ In conclusion, data from Ruggeri et $\mathrm{al}^{13}$ further validate collageninduced DDR1 activation as a critical component of pancreas remodeling and PDA progression and highlight DDR1 as an attractive target for consideration in PDA therapy.

\section{References}

1. Rahib L, Smith BD, Aizenberg R, Rosenzweig AB, Fleshman JM, Matrisian LM: Projecting cancer incidence and deaths to 2030: the unexpected burden of thyroid, liver, and pancreas cancers in the United States. Cancer Res 2014, 74:2913-2921

2. Neoptolemos JP, Kleeff J, Michl P, Costello E, Greenhalf W, Palmer DH: Therapeutic developments in pancreatic cancer: current and future perspectives. Nat Rev Gastroenterol Hepatol 2018, 15:333-348

3. Neesse A, Bauer CA, Ohlund D, Lauth M, Buchholz M, Michl P, Tuveson DA, Gress TM: Stromal biology and therapy in pancreatic cancer: ready for clinical translation? Gut 2019, 68:159-171

4. Provenzano PP, Cuevas C, Chang AE, Goel VK, Von Hoff DD, Hingorani SR: Enzymatic targeting of the stroma ablates physical barriers to treatment of pancreatic ductal adenocarcinoma. Cancer Cell 2012, 21:418-429

5. Anderson KG, Stromnes IM, Greenberg PD: Obstacles posed by the tumor microenvironment to $\mathrm{T}$ cell activity: a case for synergistic therapies. Cancer Cell 2017, 31:311-325

6. Huang $\mathrm{H}$, Wright $\mathrm{S}$, Zhang J, Brekken RA: Getting a grip on adhesion: cadherin switching and collagen signaling. Biochim Biophys Acta Mol Cell Res 2019, 1866:118472

7. Torphy RJ, Wang Z, True-Yasaki A, Volmar KE, Rashid N, Yeh B, Anderson JM, Johansen JS, Hollingsworth MA, Yeh JJ, Collisson EA: Stromal content is correlated with tissue site, contrast retention, and survival in pancreatic adenocarcinoma. JCO Precis Oncol 2018, 2018

8. Koay EJ, Lee Y, Cristini V, Lowengrub JS, Kang Y, Lucas FAS, et al: A visually apparent and quantifiable CT imaging feature identifies biophysical subtypes of pancreatic ductal adenocarcinoma. Clin Cancer Res 2018, 24:5883-5894

9. Ozdemir BC, Pentcheva-Hoang T, Carstens JL, Zheng X, Wu CC, Simpson TR, Laklai H, Sugimoto H, Kahlert C, Novitskiy SV, De Jesus-Acosta A, Sharma P, Heidari P, Mahmood U, Chin L, Moses HL, Weaver VM, Maitra A, Allison JP, LeBleu VS, Kalluri R: Depletion of carcinoma-associated fibroblasts and fibrosis induces immunosuppression and accelerates pancreas cancer with reduced survival. Cancer Cell 2014, 25:719-734

10. Leitinger B, Hohenester E: Mammalian collagen receptors. Matrix Biol 2007, 26:146-155

11. Vogel WF, Aszodi A, Alves F, Pawson T: Discoidin domain receptor 1 tyrosine kinase has an essential role in mammary gland development. Mol Cell Biol 2001, 21:2906-2917
12. Labrador JP, Azcoitia V, Tuckermann J, Lin C, Olaso E, Manes S, Bruckner K, Goergen JL, Lemke G, Yancopoulos G, Angel P, Martinez C, Klein R: The collagen receptor DDR2 regulates proliferation and its elimination leads to dwarfism. EMBO Rep 2001, 2:446-452

13. Ruggeri JM, Franco-Barraza J, Sohail A, Zhang Y, Long D, Pasca di Magliano M, Cukierman E, Fridman R, Crawford HC: Discoidin domain receptor 1 (DDR1) is necessary for tissue homeostasis in pancreatic injury and pathogenesis of pancreatic ductal adenocarcinoma. Am J Pathol 2020, 190:1735-1751

14. Shintani Y, Fukumoto Y, Chaika N, Svoboda R, Wheelock MJ, Johnson KR: Collagen I-mediated up-regulation of $\mathrm{N}$-cadherin requires cooperative signals from integrins and discoidin domain receptor 1 . J Cell Biol 2008, 180:1277-1289

15. Huang H, Svoboda RA, Lazenby AJ, Saowapa J, Chaika N, Ding K, Wheelock MJ, Johnson KR: Up-regulation of N-cadherin by collagen I-activated discoidin domain receptor 1 in pancreatic cancer requires the adaptor molecule Shc1. J Biol Chem 2016, 291:23208-23223

16. Aguilera KY, Huang H, Du W, Hagopian MM, Wang Z, Hinz S, Hwang TH, Wang H, Fleming JB, Castrillon DH, Ren X, Ding K, Brekken RA: Inhibition of discoidin domain receptor 1 reduces collagen-mediated tumorigenicity in pancreatic ductal adenocarcinoma. Mol Cancer Ther 2017, 16:2473-2485

17. Zhu D, Huang H, Pinkas DM, Luo J, Ganguly D, Fox AE, Arner E, Xiang Q, Tu ZC, Bullock AN, Brekken RA, Ding K, Lu X: 2-Amino2,3-dihydro-1H-indene-5-carboxamide-based discoidin domain receptor 1 (DDR1) inhibitors: design, synthesis, and in vivo antipancreatic cancer efficacy. J Med Chem 2019, 62:7431-7444

18. Ambrogio C, Gomez-Lopez G, Falcone M, Vidal A, Nadal E, Crosetto N, Blasco RB, Fernandez-Marcos PJ, Sanchez-Cespedes M, Ren X, Wang Z, Ding K, Hidalgo M, Serrano M, Villanueva A, Santamaria D, Barbacid M: Combined inhibition of DDR1 and Notch signaling is a therapeutic strategy for KRAS-driven lung adenocarcinoma. Nat Med 2016, 22:270-277

19. Gao H, Chakraborty G, Zhang Z, Akalay I, Gadiya M, Gao Y, Sinha S, Hu J, Jiang C, Akram M, Brogi E, Leitinger B, Giancotti FG: Multiorgan site metastatic reactivation mediated by non-canonical discoidin domain receptor 1 signaling. Cell 2016, 166:47-62

20. Takai K, Drain AP, Lawson DA, Littlepage LE, Karpuj M, Kessenbrock K, Le A, Inoue K, Weaver VM, Werb Z: Discoidin domain receptor 1 (DDR1) ablation promotes tissue fibrosis and hypoxia to induce aggressive basal-like breast cancers. Genes Dev 2018, 32:244-257

21. Selfors LM, Stover DG, Harris IS, Brugge JS, Coloff JL: Identification of cancer genes that are independent of dominant proliferation and lineage programs. Proc Natl Acad Sci U S A 2017, 114: E11276-E11284

22. Wollny D, Zhao S, Everlien I, Lun X, Brunken J, Brune D, Ziebell F, Tabansky I, Weichert W, Marciniak-Czochra A, Martin-Villalba A: Single-cell analysis uncovers clonal acinar cell heterogeneity in the adult pancreas. Dev Cell 2016, 39:289-301

23. Avivi-Green C, Singal M, Vogel WF: Discoidin domain receptor 1deficient mice are resistant to bleomycin-induced lung fibrosis. Am J Respir Crit Care Med 2006, 174:420-427

24. Flamant M, Placier S, Rodenas A, Curat CA, Vogel WF, Chatziantoniou C, Dussaule JC: Discoidin domain receptor 1 null mice are protected against hypertension-induced renal disease. J Am Soc Nephrol 2006, 17:3374-3381

25. Gao M, Duan L, Luo J, Zhang L, Lu X, Zhang Y, Zhang Z, Tu Z, Xu Y, Ren X, Ding K: Discovery and optimization of 3-(2-(pyrazolo [1,5-a]pyrimidin-6-yl)ethynyl)benzamides as novel selective and orally bioavailable discoidin domain receptor 1 (DDR1) inhibitors. J Med Chem 2013, 56:3281-3295

26. Zolghadri Y, Pal Choudhuri S, Ocal O, Layeghi-Ghalehsoukhteh S, Berhe F, Hale MA, Wilkie TM: Malnutrition in pancreatic ductal adenocarcinoma (PDA): dietary pancreatic enzymes improve shortterm health but stimulate tumor growth. Am J Pathol 2018, 188: $616-626$ 